Série Éléments de sûreté nucléaire, de radioprotection et de sécurité

\title{
Éléments de sécurité et de non-prolifération
}

\author{
Jean Jalouneix
}


Illustration de couverture : mosaïque de photographies relatives aux sujets traités dans les différents tomes de la série Éléments de sûreté nucléaire, de radioprotection et de sécurité. Mise en avant, détourées en jaune, de celles illustrant les thèmes développés dans ce premier tome de la série.

Imprimé en France

ISBN : 978-2-7598-1676-7

DOI: 10.1051/978-2-7598-1676-7

Tous droits de traduction, d'adaptation et de reproduction par tous procédés, réservés pour tous pays. La loi du 11 mars 1957 n'autorisant, aux termes des alinéas 2 et 3 de l'article 41, d'une part, que les « copies ou reproductions strictement réservées à l'usage privé du copiste et non destinées à une utilisation collective », et d'autre part, que les analyses et les courtes citations dans un but d'exemple et d'illustration, " toute représentation intégrale, ou partielle, faite sans le consentement de l'auteur ou de ses ayants droit ou ayants cause est illicite » (alinéa $1^{\mathrm{er}}$ de l'article 40 ). Cette représentation ou reproduction, par quelque procédé que ce soit, constituerait donc une contrefaçon sanctionnée par les articles 425 et suivants du code pénal.

(c) IRSN 2015 